\title{
Left Atrial Myxoma as the Cause of Syncope in an Adolescent
}

\author{
Danilo Castellani Nogueira, Delio Bontempo, Antonio Carlos Menardi, Walter Vilella de A. Vicente, \\ Paulo J osé de Freitas Ribeiro, Paulo Roberto Barbosa Evora
}

Ribeirão Preto, SP - Brazil

A case of left atrial myxoma is reported in a 14-yearold female patient with recurrent episodes of syncope. The patient was immediately referred to the cardiologist after an inconclusive neurological investigation. Syncope is a symptom thoroughly described as being associated with heart myxomas, but its specific association with adolescence is extremely rare. The authors discuss the clinical manifestations of the disease, emphasizing the diagnostic difficulties on the basis of the unspecific symptoms. As part of the analysis a Brazilian literature review was carried out. Also, the authors experience in the surgical treatment of the heart tumors is briefly presented.

In agreement with classical data from the Institute of Pathology of the Armed Forces (Washington, DC), primary tumors of the heart, benign or malign, are found in $0.001 \%-0.28 \%$ of all of autopsies, and the myxomas represent $50 \%$ of the benign tumors of the heart ${ }^{1}$. Clinical experiences at two of the largest Brazilian institutions (InCorUSP and Dante Pazzanese Institute of Cardiology) demonstrate the wide prevalence of the heart myxomas as primary heart tumors. At InCor-USP, in a series of 50 patients treated over a period of 18 years, $84 \%$ of the myxomas were of the histological type ${ }^{2}$. At the Dante Pazzanese Institute of Cardiology, the experience was similar. Over a period of 28 years, with a series of 52 patients with heart tumors, $82.7 \%$ of the tumors were myxomas ${ }^{3}$.

At the Ribeirão Preto Heart Hospital, over a period of 23 years, surgeons at the CECORP - Ribeirão Preto Specialized Heart and Lung Center operated on 7 patients for removal of heart tumors, 6 patients with myxomas and 1 patient with leiomyosarcoma metastasis. Among the 6 patients with heart myxomas, the age varied from 14 to 78 years, 4 were females, and all tumors were located at the left atrium.

Ribeirão Preto Heart Hospital/Waldemar B. Pessoa Foundation, CECORP Ribeirão Preto Specialized Center of Heart and Lung, and UNAERP Medical School of Ribeirão Preto.

Mailing address: Paulo Roberto B. Evora - Rua Rui Barbosa, 367/15 - 14015-120 Ribeirão Preto, SP, Brazil - E-mail: prbevora@keynet.com.br
In a period of more than 50 years (1949 to 2001), 20 articles about heart tumors have been published in the Brazilian Archives of Cardiology (Source: Medline and Index Medicus), 5 articles have been published in the Brazilian Journal of Cardiovascular Surgery (Source: Scielo), and 13 other articles have been published in other Brazilian journals (Source: Bireme). It is possible to estimate that around 270 cases have been discussed in the Brazilian literature based on these 3 cumulative medical reference indexes.

Although syncope is a symptom thoroughly described, resulting from myxoma left ventricular inflow obstruction, its incidence in the adolescent is rare, creating difficulties for the clinical evaluation of syncope in this age group. A unique case was reported in the literature of syncope as a consequence of left ventricle outflow obstruction, caused by a myxoma localized in this cardiac chamber ${ }^{4}$. A case of ischemic stroke in an 8-year-old female child has been reported in the Brazilian literature 5 .

These data justify this case report of syncope in an adolescent caused by the presence of a left atrial heart myxoma that intermittently obstructed the mitral valve.

\section{Case Report}

A 14-year-old female, resident of São Simão (SP), had a clinical cardiology appointment in April 2000 because of 3 episodes of syncope over the previous 3 months. During this interval of time, she was evaluated by a neurologist, without any diagnostic conclusion about the syncope. She was then directed to the cardiologist due to the presence of palpitations experienced at rest.

During the first cardiology consultation, a syncope diagnosis was the main focus of the investigation. The clinical anamnesis revealed palpitations, a heart rate of $130 \mathrm{bpm}$, and low corporal weight with accentuated loss in the prior 3 months. The heart auscultation, by this time, did not show evidence of a heart murmur. With the clinical hypothesis of hyperthyroidism, thyroid hormone dosages and an electrocardiogram were requested. The hormonal function was normal. The electrocardiogram identified a sinus tachycardia and a possible left atrium enlargement. The diagnostic 
hypothesis of a cardiopathy with left atrial overload was assumed, and a chest $\mathrm{x}$-ray and a transthoracic echocardiogram were requested. The $\mathrm{x}$-ray showed a congested pulmonary hilus, increased density in the topography of the left atrium, and evident prominence of the main pulmonary artery. Doppler echocardiography demonstrated moderate left atrium dilation, with a suggestive image of an intracardiac myxoma. A mitral valve insufficiency, caused by the tumor prolapse inside of the left ventricle through this valve (fig.1-B) was also documented. After these examinations, heart surgery was scheduled for the patient.

The patient experienced a new episode of syncope. During a venous blood sample collection, the patient experienced another prodrome of the symptom as she sat down. With the increase in anemia, the patient began to experience great fatigue on exertion and lost 9 kilos in 4 months. A constant cough began to occur when the patient was lying down, and the child indicated that in certain positions she did not have dyspnea and slept very well in the ventral decubitus position. A family heart tumor antecedent was not identified. The heart auscultation revealed a mitral systolic murmur that changed its characteristics according to the decubitus position. Her blood pressure was $90 / 60 \mathrm{mmHg}$, the heart rate was $100 \mathrm{bpm}$, and congestive signs were not present. The preoperative examinations showed hemoglobin of $9.1 \mathrm{~g} / \mathrm{dL}$, hematocrit of $32 \%, 7400$ leucocytes $/ \mathrm{dL}$, 54,8000 platelets $/ \mathrm{mm}^{3}$, creatinine $0.6 \mathrm{~g} / \mathrm{dL}$, urea $18 \mathrm{~g} / \mathrm{dL}$, and urine tests without abnormalities.

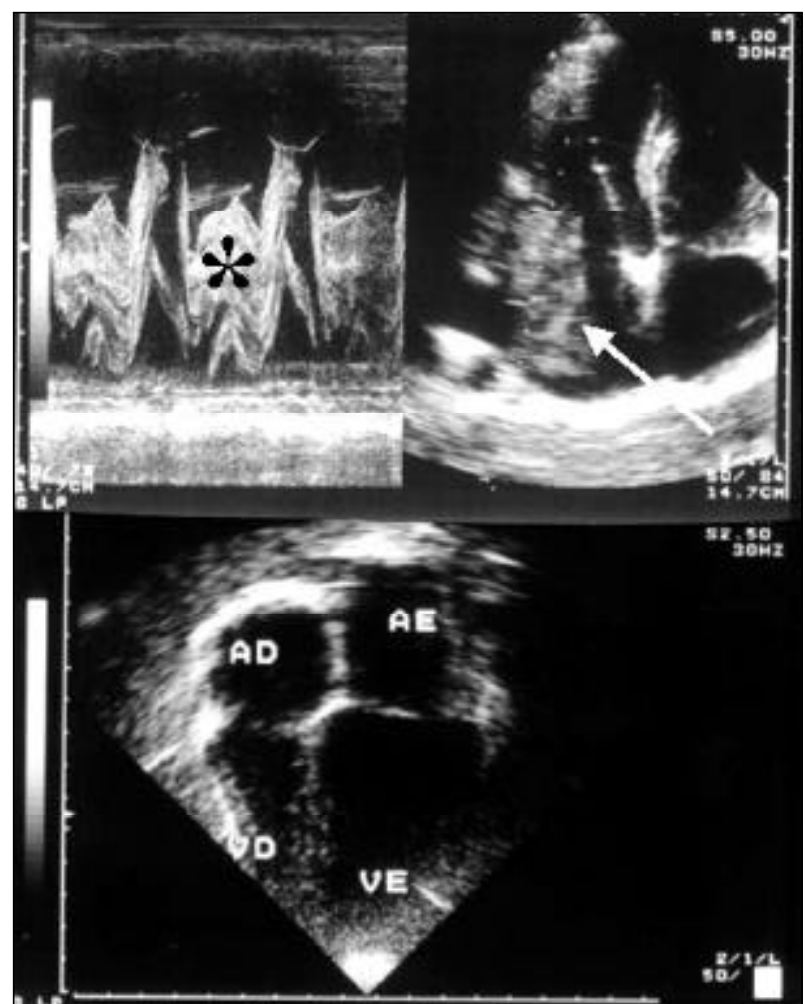

Fig. 1 - Echocardiography. A) The M-mode, asterisk denotes echodense images of the tumor; B) two-dimensional "four chamber" echocardiogram, arrow denotes the prolapsed tumor through the mitral valve; C) two-dimensional "four chamber" showing the postoperative result without the tumoral image.
She underwent the surgical procedure with the aid of cardiopulmonary bypass in May 2000 through a median sternotomy. Tense and increased right heart cavities were observed. The cardiotomy consisted of a transverse incision of the right and left atriums, extended about $2 \mathrm{~cm}$ toward the interatrial septum. A big encapsulated tumor almost filling the whole left atrium was observed. It was pedunculated between the inferior pulmonary vein and the inferior vena cava. The tumor was excised with a good margin around its pedicle, followed by a direct atrial suture. The anatomico-pathological diagnosis confirmed a heart myxoma(fig. 2).

In 1 year, the patient regained 11 kilos, no longer experienced syncopes and palpitations, and her anemia completely disappeared. She has been absolutely symptom free in 20 months of follow-up.

\section{Discussion}

Heart tumors are not very common pathologies observed in clinical practice. Secondary tumors are more frequent, in a ratio that can reach 40:1. Primary heart tumors are rare, usually benign in nature, and myxomas are responsible for $50 \%$ of the cases. Heart myxomas are more prevalent in women and are rare in children. In 94\% of patients, lone tumors are found located mainly in the left atrium, with an incidence of up to $70 \%$. Myxomas most commonly occur between the third and the sixth decade of life. They do not have a familiar pattern of characteristics, except for familial myxoma that has autosomal dominant inheritance. The myxomas are round or oval, compact, sessile, or pedunculated. The most frequent are pedunculated myxomas, and sometimes represent $83 \%$ of cases. They appear in the endocardium, starting from a mesenchymal or multipotent subendocardial cells, presenting as a white, yellowish, or brownish mass, frequently covered by thrombi. The tumors are, on average, 5 -cm in diameter and weight from 50 to 60 grams.

In spite of the benign characteristics, the symptoms will depend on the size, form, mobility, and location of the tumor. Dyspnea is the most common manifestation in up to

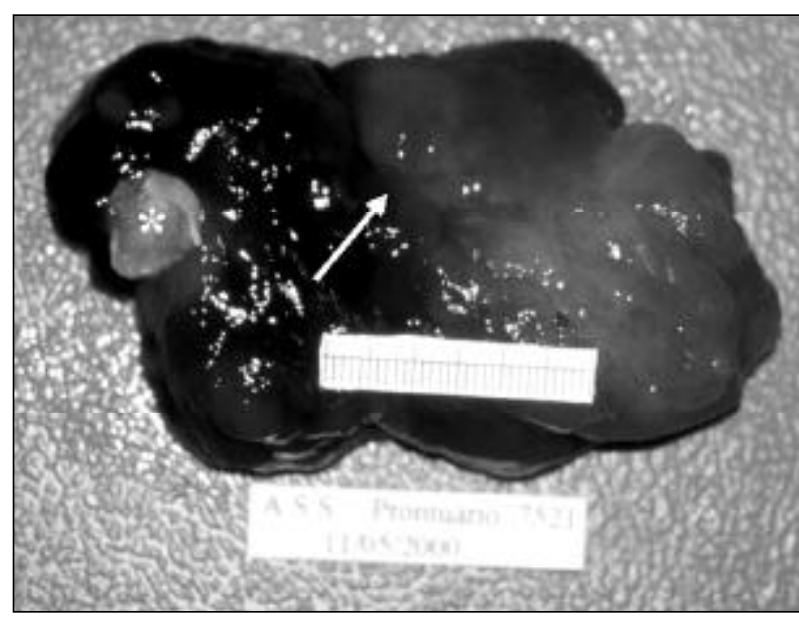

Fig. 2 - Myxoma surgically excised with cardiopulmonary bypass support. The arrow denotes the mitral valve ring impression and the asterisk denotes the tumor pedicle. 
$80 \%$ of patients, and congestive heart failure appears in $56 \%$ of them. The patients can present with atypical chest pain, syncope, lethargy, indisposition, weight loss, palpitations, peripheral edema, acute pulmonary edema, lung embolism, cerebral ischemia, transitory ischemic attack, atrial fibrillation, atrial flutter and other tachyarrhythmias, hemoptysis, systemic embolizations and infection with clinical signs of bacterial endocarditis. The heart auscultation can be quite similar to that of mitral valve disease, and may be associated with a tumoral sound (tumoral plop). The most useful examination in the diagnosis is the echocardiogram that is highly sensitive and can diagnose up to $100 \%$ of the cases. Some nonspecific data can be observed, such as elevated sedimentation rates, anemia, leukocytosis, thrombocytopenia, thrombocytosis, elevated immunoglobulin concentration, and increased $\mathrm{C}$ reactive protein. The myxomas should be included in the differential diagnosis of valvular heart disease, cardiac failure, cardiomegaly, bacterial endocarditis, supraventricular and ventricular rhythm disturbances, syncope, lung and systemic embolism. The secondary heart tumors should be investigated through laboratory inflammatory proteins, increase in the sedimentation rate, anemia, abnormal electrophoresis, hypoproteinemia, and elevation of alkaline phosphatase.

In a Medline literature metaanalysis, we selected 295 patients with myxoma, including 68\% (202 patient) female and $32 \%$ (93 patient) male. The myxomas localized in the left atrium reached $81 \%$ (239 patients), $16 \%$ (47 patients) in the right atrium, and $3 \%$ (9 patients) in another location. The average age was 48 years and $92.7 \%$ were symptomatic. The clinical manifestations had variations in the several studies. Dyspnea was the predominant symptom. Other phenomena mentioned frequently in the literature are embolism varying from $17 \%$ to $50 \%$, tachyarrhythmias from $24 \%$ to $25.7 \%$, constitutional symptoms from $9.3 \%$ to $45 \%$ that were present only in left atrial myxoma, chest pain from $12.9 \%$ to $25 \%$, and syncope from $6 \%$ to $18.9 \%$.

Heart myxomas might be included in a differential diagnosis of heart valve disease, heart failure, cardiomegaly, bacterial endocarditis, heart arrhythmias, syncope, and systemic and pulmonary embolism. A laboratory clinical analysis may include inflammatory proteins, elevated sedimentation rates, anemias, abnormal electrophoresis, hypoproteinemia, and increased levels of alkaline phosphatase.

In the clinical evaluation of patients with heart syncope, the electrocardiogram and echocardiogram are fundamental for the diagnosis of heart arrhythmias and valvar diseases. The heart tumors are usually diagnosed by ultrasound images. Other methods can aid in the myxoma diagnosis, such as computerized tomography and magnetic resonance imaging, and they can be important to guide the surgical approach. Cineangiocoronariography can be a valuable examination in the detection of the tumors and has been in some studies evident in $84 \%$ of cases. The complete preoperative heart evaluation is mandatory to instruct the surgeon about other heart problems like valvoplasty or artificial valve prosthesis and atrial chamber reconstruction.

The surgical intervention with complete resection of the tumor is essential for the cure of the disease, and it prevents tumor recurrence with subsequent reoperations that expose the patient to other complications, such as bleeding and the need for hemoderivatives ${ }^{6-8}$. The tumor recurrence rate is very low, and the survival rate for surgery is very good, with hospital mortality around $4 \%$. One of the CECORP cases was treated for a tumor recurrence. An interesting Brazilian literature case report reveals a myxoma recurrence with a multicavity location. According to a few studies, tumor recurrence can be linked to the production of growth factors. Other possible recurrence mechanisms include incomplete excision of the tumor, intramyocardial implantation, peripheral embolizations, and growth starting from other foci. However, in a general way, the surgical result in the long-term follow-up is very good, with a few symptomatic patients remaining in functional class I of the New York Heart Association (NYHA) and a minority in functional class II.

The patient, whose case was reported in this paper, experienced heart syncope caused by a myxoma, that is responsible for 2 to $3 \%$ of the syncopes under medical evaluation. The decrease in cardiac output due to the tumoral obstruction is responsible for the syncopes. This symptom is more common after periods of short physical activity and is reversed as soon as the obstruction is removed. The obstruction, mainly caused by big pedunculated tumors, can decrease cerebral flow and lead to sudden death.

The patients with heart myxoma generally have a classic triad of symptoms that include constitutional, embolic, or obstructive manifestations. In the present case, the adolescent did not have embolic phenomena. The constitutional manifestations many times remind autoimmune diseases. To explain the anemia and the accentuated weight loss, which disappeared with the tumor excision, it is not possible to find any specific mechanism. It is unknown whether the tumor secretes some substance or whether it links with some immunologic mechanism similar to the autoimmune disease physiopathology. Another outstanding detail in this case report is the young age of the patient. These data could suggest a congenital characteristic; however, a careful investigation of the family antecedents did not reveal any similar familial case. At any circunstance, clinicians should be alert for the possibility of left heart myxoma as a cause of syncope, emphasizing its rarity in children and adolescents. 


\section{References}

1. Mc Allister HA Jr, Fenoglio JJ Jr. Tumors of the cardiovascular system. Fascicle 15, Second Series. Atlas of Tumor Pathology. Washington DC: Armed Forces Institute of Pathology, 1978.

2. Fernandes F, Soufen HN, Ianni BM, Arteaga E, Ramires FJ, Mady C. Primary neoplasms of the heart: clinical and histological presentation of 50 cases. Arq Bras Cardiol 2001; 76: 231-7.

3. Abdulmassih CN, Salerno PR, Dinkhuysen JJ, et al. Correção cirúrgica dos tumores primários do coração. Rev Bras Cir Cardiovasc 1992; 7: 145-52.

4. Delgado LJ, Montiel J, Guindo J, et al. Myxoma of the left ventricle: a cause of syncope in an adolescent. Rev Esp Cardiol 1998; 51: 674-6.
5. Takakura IT, Godoy MF, Soares MJ, Moscardini AC, Braile DM. Mixoma atrial esquerdo com acidente vascular cerebral isquêmico em criança. Arq Bras Cardiol 1998; 71: 135-7.

6. Reynen K. Cardiac myxomas. N Engl J Med 1995; 333: 1610-7.

7. Husar J, Ivancan V, Korda Z, et al. Cardiac myxoma: diagnostic approach, surgical treatment and follow up: a twenty years experience. J Cardiovasc Surg 1996; 37 : 113-7.

8. Lijoi A, Scoti P, Faveto C, et al. Surgical management of intracardiac myxomas. A 16-year experience. Tex Heart Inst J 1993; 20: 231-4.

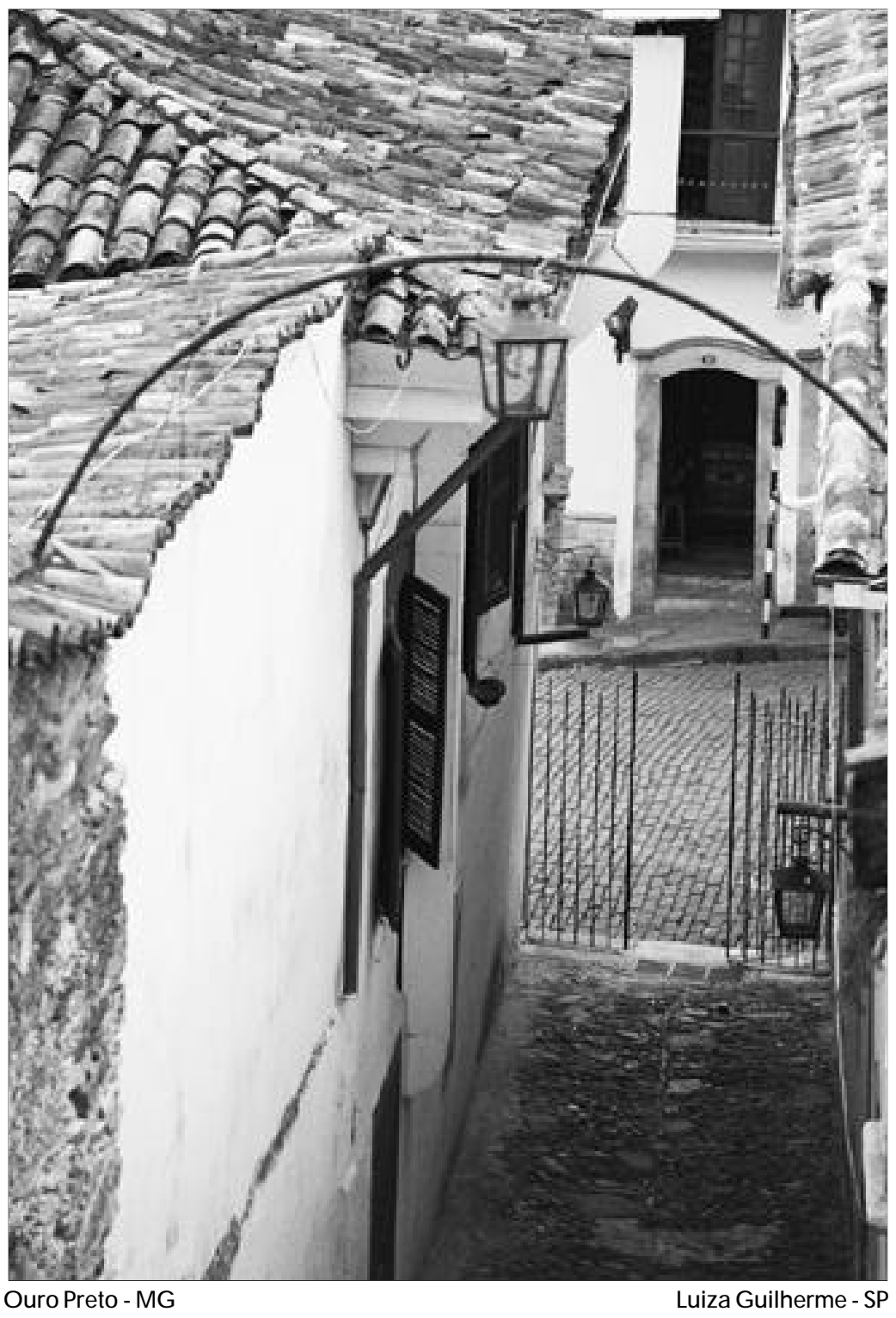

Editor da Seção de Fotografias Artísticas: Cícero Piva de Albuquerque

Correspondência: InCor - Av. Dr. Enéas C. Aguiar, 44 - 05403-000 - São Paulo, SP - E-mail: delcicero@incor. usp.br 